\title{
Energy Efficient Hierarchical Unequal Clustering in Wireless Sensor Networks
}

\author{
B. Baranidharan ${ }^{1 *}$, S. Srividhya ${ }^{2}$ and B. Santhi ${ }^{1}$ \\ 'Department of Computer Science Engineering, SASTRA University, India; \\ baranidharan@it.sastra.edu, shanthi@cse.sastra.edu \\ 2Advanced Computing, SASTRA University, India; swaminathan_srividhya@yahoo.com
}

\begin{abstract}
Energy efficient modeling is a major issue in the wireless sensor network. The main solution for energy efficient routing is by means of clustering. This paper proposes an unequal clustering approach in the networks for even energy distribution. It also reduces the overall energy consumption which in turn improves the network lifetime. The simulation is carried out in MATLABR2010a. The energy needed for entire operations for one round using the proposed method is lesser than that of LEACH, an equal clustering methodology.
\end{abstract}

Keywords: Clustering, Energy Efficient, Unequal Clustering

\section{Introduction}

Wireless Sensor Networks are extremely dispersed networks of small, light weight Wireless Sensor Nodes (SN). The network can be classified as homogeneous or heterogeneous type. The homogenous network is built upon with the nodes of same capability, whereas in heterogenous of different capable nodes. Mainly these sensors are used to observe the environment based on the desired physical parameters. Some of the real time applications of WSN are climate monitoring, solar monitoring, power plant monitoring, industrial measurements, and resource monitoring and event detections.

Most of the WSNs have been designed in such a way that the nodes sensing the environment transmit its generated data to the Central BS (BS). In this case, energy consumption is high when all nodes communicate directly with the BS. Clustering promotes a way of transmitting data to the BS using multiple hops ${ }^{1}$. In clustering, $\mathrm{CH}$ nodes $(\mathrm{CH})$ collect the information from its members and aggregate it. Only $\mathrm{CH}$ nodes forwards those aggregated information to the BS. This approach definitely mitigates the overall energy consumption.

In general, clustering ${ }^{2}$ can be classified into equal and unequal clustering. Equal clustering ensures the same number of cluster members for all the clusters. All $\mathrm{CH}$ nodes communicate with the BS in a multi-hop manner through other $\mathrm{CH}$ nodes. The $\mathrm{CH}$ nearer to the BS finally relay all the gathered information to the BS. In this case there may be a chance for those relay $\mathrm{CH}$ nodes to die early. To avoid this situation unequal clustering has been proposed.

The paper is organized as follows: In section 2, works related to unequal clustering is briefly enlightened. In section 3, the proposed algorithm is explained in detail. In section4, the proposed protocol HUCA is compared with LEACH. The comprehensive evaluation outcomes are mentioned.

\section{Related Works}

There are several clustering methodologies have been proposed for wireless sensor networks. Some of them are discussed below:

\subsection{LEACH}

$\mathrm{LEACH}^{3}$ utilizes an equal clustering methodology. The network is divided into Clusters. LEACH elects the $\mathrm{CH}$ node based on local decisions. $\mathrm{CH}$ has been chosen

*Author for correspondence 
at random based on some threshold value. Based on the threshold on every round the $\mathrm{CH}$ nodes have been chosen. The $\mathrm{CH}$ node collects the information from the ordinary nodes in its cluster. $\mathrm{CH}$ aggregates the data before forwarding to BS. In $\mathrm{LEACH}$, there may be a chance of selecting a lower energy node as $\mathrm{CH}$ nodes.

\subsection{HEED}

Hierarchical Energy Efficient Distributed Clustering ${ }^{4}$ considers the remaining energy level of SNs for the stochastic selection of $\mathrm{CH}$ nodes. The degree of the $\mathrm{CH}$ nodes or average distance to its nearby neighbor nodes is used to conclude the cluster-head when two nodes are competing for $\mathrm{CH}$. HEED provides a better performance than LEACH.

\subsection{LCC}

The improved version of LEACH is LEACH-Centralized with Chain (LCC). LCC $^{5}$ forms a super cluster with the $\mathrm{CH}$ nodes as its members. $\mathrm{CH}$ nodes are selected by the same procedure as LEACH. This protocol chooses one leader among super cluster members. By means of greedy approach, it builds a chain connection between the super cluster members to BS via the leader. Super CH Leader aggregates the data from $\mathrm{CH}$ nodes. Finally, the super $\mathrm{CH}$ Leader sends the aggregated data to the BS directly.

\subsection{EEGTP}

In Energy Efficient Graph Theory Protocol ${ }^{6}$ for WSN, all the $\mathrm{CH}$ nodes communicate with the BS in multi hop manner. The last node in the multi hop communication named super aggregator gathers all the data from $\mathrm{CH}$ nodes and finally send it to the BS.

\subsection{CHEF}

$\mathrm{CH}$ nodes Election using Fuzzy logic is an unequal clustering protocol ${ }^{7}$ which selects the cluster in a centralized manner. BS is involved in the selection process. This protocol uses three fuzzy descriptors such as node concentration, residual energy and node centrality for electing the $\mathrm{CH}$. BS creates fuzzy rules and computes the crisp output representing the chance for $\mathrm{CH}$.

\subsection{EECDC}

Energy Efficient Coverage Aware Data Collection ${ }^{8}$ in Wireless Sensor Networks first forms the MIS and then selects the effective $\mathrm{SH}$. Communication is divided into inter and intra setups. Intercommunication is handled by using multi hop between SH to BS.

\subsection{EEUC}

Energy Efficient Unequal Clustering ${ }^{9}$ uses a probabilistic approach to choose the tentative $\mathrm{CH}$. Tentative $\mathrm{CH}$ nodes participate in the $\mathrm{CH}$ competition. EEUC considers the node's residual energy for $\mathrm{CH}$ selection.

\subsection{EAUCF}

Energy Aware Unequal Clustering using Fuzzy logic ${ }^{10}$ deals with unequal clustering uses multi hop routing. Unequal clustering delays the first node die time when compared to equal clustering used in LEACH. Fuzzy rules are utilized for selecting the coverage distance of $\mathrm{CH}$ nodes based on three input variable distances to the BS, residual energy and node degree. Moreover it combines the advantages of unequal clustering and fuzzy logic approaches.

\subsection{IFUC}

In the protocol Improved Fuzzy Unequal Clustering ${ }^{11}$, the input fuzzy descriptors for each node are its distance to BS, energy level and local density. The probability of nodes becoming $\mathrm{CH}$ and its radius is calculated as output fuzzy parameters using if- then rules. After that, ACO technique is to produce the energy-efficient route between $\mathrm{CH}$ nodes and $\mathrm{BS}$ in multi hop manner.

\subsection{DSBCA}

Uniform clustering may lead to unbalanced load for $\mathrm{CH}$ nodes nearer to the BS because of load due to relaying. To balance the load, Distributed Self Organization Load Balanced Clustering Algorithm ${ }^{12}$ helps to provide a non uniform clustering. Hierarchical arrangement of clusters is created. The distance from the BS and connectivity density is used to calculate the coverage radius of each $\mathrm{CH}$. The nodes within the coverage radius become members of that cluster.

\section{HUCA Algorithm Preliminaries}

For the configuration of the network, the preliminaries are defined below:

- All nodes are stationary after deployment.

- All nodes know its location using GPS receivers.

- The network is of homogeneous type. 
- All SNs have same initial energy.

- The BS is located away from the field area to be monitored.

\subsection{Radio Model}

The free space radio model is used to calculate the energy in free space. Eq. 1 characterizes the amount of energy needed for forwarding 'l' bit of data to a node at a distance d. Eq. 2 stands for the energy needed to receive 'l' bit of data.

$$
\begin{aligned}
& \mathrm{E}_{\mathrm{tx}}(1, \mathrm{~d})=\mathrm{l}^{*} \mathrm{E}_{\text {ele }}+\mathrm{l}^{*} \mathrm{e}_{\mathrm{fs}} \mathrm{d}^{2} \\
& \mathrm{E}_{\mathrm{rx}}(\mathrm{l})=\mathrm{l}^{*} \mathrm{E}_{\mathrm{ele}} \\
& \mathrm{E}_{\text {ele }} \rightarrow \text { Energy Consumption/bit in transceiver circuit. } \\
& \mathrm{e}_{\mathrm{fs}} \rightarrow \text { Energy degenerated/bit in RF amplifier }
\end{aligned}
$$

\subsection{Proposed Algorithm}

The proposed algorithm is as follows: Hierarchical Unequal Clustering Algorithm (HUCA) is based on unequal clustering. It uses the local knowledge to form clusters and selecting the $\mathrm{CH}$ nodes. HUCA is divided into three phases. They are,

i. Grid formation

ii. $\mathrm{CH}$ node election

iii. Data collection

\subsubsection{Grid Formation}

Network area is separated into three horizontal grids based on the longitudinal distance from the BS. The grid located near to the $\mathrm{BS}$ will be having more number of $\mathrm{CH}$ nodes which guarantees the uniform energy consumption among the $\mathrm{CH}$ nodes. In equal clustering the nearby $\mathrm{CH}$ nodes to the $\mathrm{BS}$ involves high data traffic which costs its lifetime.

\begin{tabular}{|l|}
\hline Grid formation phase \\
\hline Network Area $=100^{*} 100$ \\
Levels $=3$ \\
$\mathrm{Y} \leftarrow$ longitudinal axis \\
If $\mathrm{Y}</ 3$ then \\
$\mathrm{L} 1 \leftarrow$ for all $\mathrm{Ni}(\mathrm{X}, \mathrm{Y})$ \\
Else if $\mathrm{Y}<2^{*}(100 / 3)$ then \\
$\mathrm{L} 2 \leftarrow$ for all $\mathrm{Ni}(\mathrm{X}, \mathrm{Y})$ \\
Else $\quad \mathrm{L} 3 \leftarrow$ for all $\mathrm{Ni}(\mathrm{X}, \mathrm{Y})$
\end{tabular}

\subsubsection{CH Nodes Selection Phase}

Since the network is homogeneous in nature the initial $\mathrm{CH}$ node selection will be a random selection. Each node will choose a number in the range 0 to 1 . The node having the highest value among its neighbor will elect itself as the $\mathrm{CH}$ node and broadcast its selection to the nearby nodes. After receiving this selection messages from one or more $\mathrm{CH}$ nodes, the ordinary nodes join the nearby $\mathrm{CH}$ node. In the next $\mathrm{CH}$ node selection phase the node having the higher remaining residual energy will be elected as the $\mathrm{CH}$ nodes.

\begin{tabular}{c} 
CH nodes election \\
\hline For $\mathrm{i}=1: 3$ \\
For $\mathrm{j}=1: \mathrm{n}$ \\
Ret number of clusters ' $\mathrm{n}$ ' in each level \\
CHlist $\leftarrow \mathrm{N}_{\mathrm{ij}}$ \\
CMlist $\leftarrow$ all nodes $\mathrm{N}$ except $\mathrm{CH}$ \\
If $\mathrm{N}_{\mathrm{ij}}=\mathrm{CM}$ then \\
Sends joinCHms $\left(\mathrm{N}_{\mathrm{ij}(\mathrm{ID})}\right)$ to nearby $\mathrm{CH}$ \\
Else \\
Accepts the joinCHmsg() from $\mathrm{N}_{\mathrm{ij}(\mathrm{ID})}$ \\
End \\
End \\
End \\
No. of nodes in each cluster vary because it \\
is based on the communication range of the \\
head.
\end{tabular}

\subsubsection{Data Collection}

The nodes transmit its data to its $\mathrm{CH}$ nodes in the respective grids. The $\mathrm{CH}$ node aggregates its member node's data and forwards it to the next grid $\mathrm{CH}$ node which is nearer to its location. Thus the $\mathrm{CH}$ nodes in the grid close to $\mathrm{BS}$ acts as router nodes to their higher level grid nodes. Also the rapid energy depletion of $\mathrm{CH}$ nodes nearby $\mathrm{BS}$ is avoided by increasing their number.

\section{Simulation and Result}

MATLAB R2010a is used to simulate the proposed algorithm HUCA. The network setup is shown in the Figure 1. Each node is identified by its unique ID. The network region is divided into 3 horizontal grids. The network parameters are stated out in Table 1. 
Energy needed for one round is calculated by the proposed algorithm. Figure 2 compares HUCA with LEACH. HUCA proves that the energy needed for one data collection round is $11 \%$ less than the energy needed for the same using LEACH.

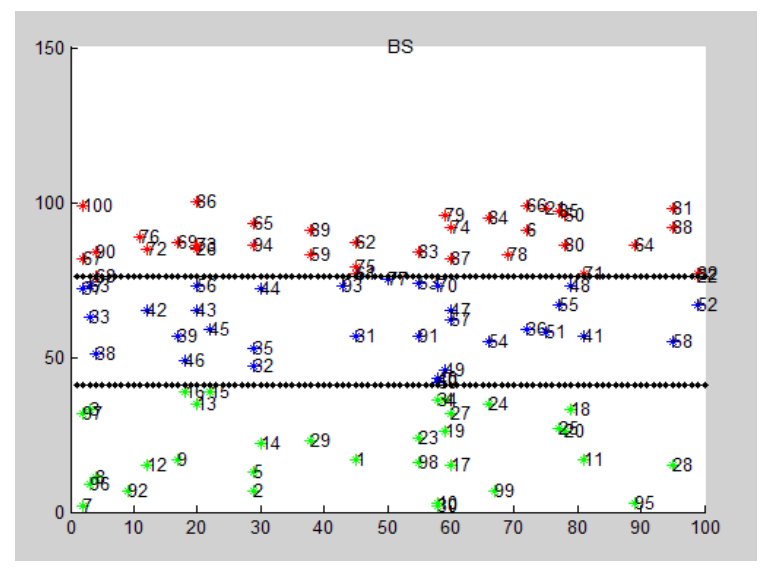

Figure 1. Wireless Sensor Network of 100 nodes.

Table 1. Simulation parameters

\begin{tabular}{ll}
\hline Parameter & Value \\
\hline Network size & $100^{*} 100 \mathrm{~m}$ \\
BS location & $(50,150)$ \\
Number of nodes & 100 \\
Packet size & $4000 \mathrm{bits}$ \\
Initial energy & $1 \mathrm{~J}$ \\
$\mathrm{E}_{\text {ele }}$ & $50 \mathrm{nJbit}^{-1}$ \\
$\mathrm{E}_{\text {fs }}$ & $10 \mathrm{pJbit}^{-1}$ \\
$\mathrm{E}_{\text {agg }}$ & $50 \mathrm{nJbit}^{-1}$ \\
\hline
\end{tabular}

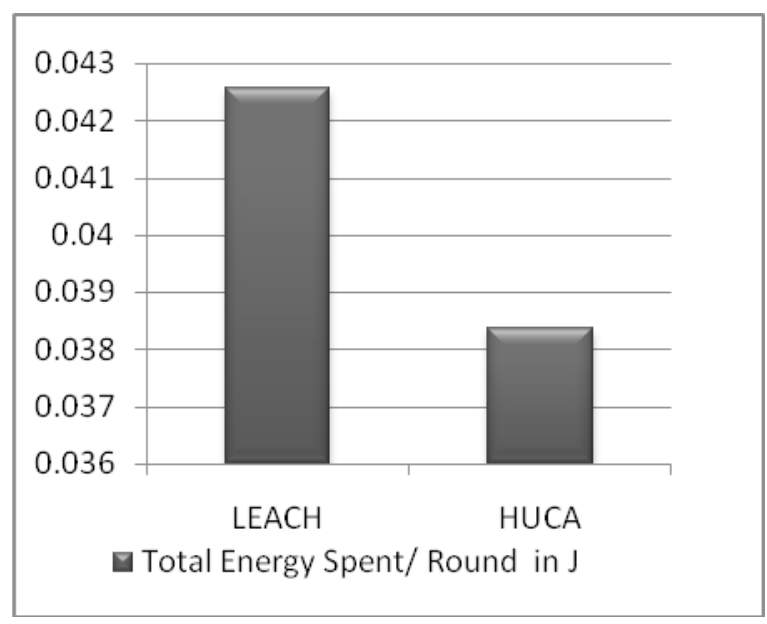

Figure 2. Comparison of LEACH and HUCA.
LEACH follows an equal clustering. Number of nodes in each and every cluster is same. This is depicted in the Figure 3. Using HUCA the above network setup is divided into 3 horizontal grids. Again it is subdivided which results in total of 16 unequal clusters. Figure 4 shows the 16 clusters and its number of nodes.

Figure 5 shows the energy consumption of 10 clusters using LEACH protocol. The energy consumption of 16 clusters in HUCA algorithm is shown in Figure 6.

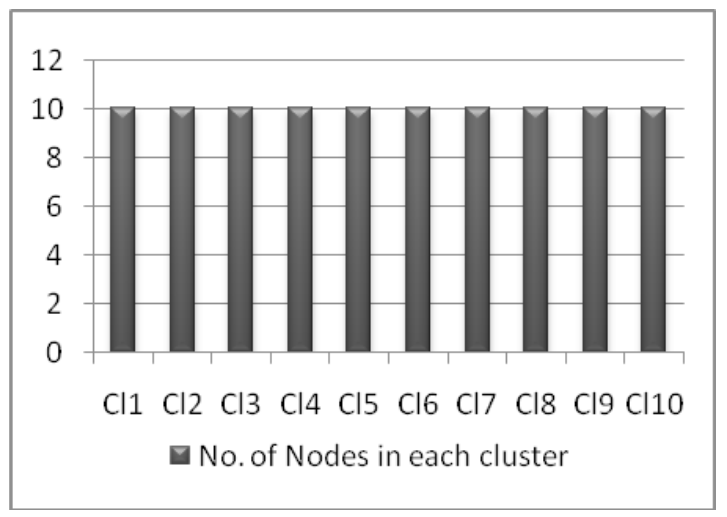

Figure 3. Equal clustering in LEACH.

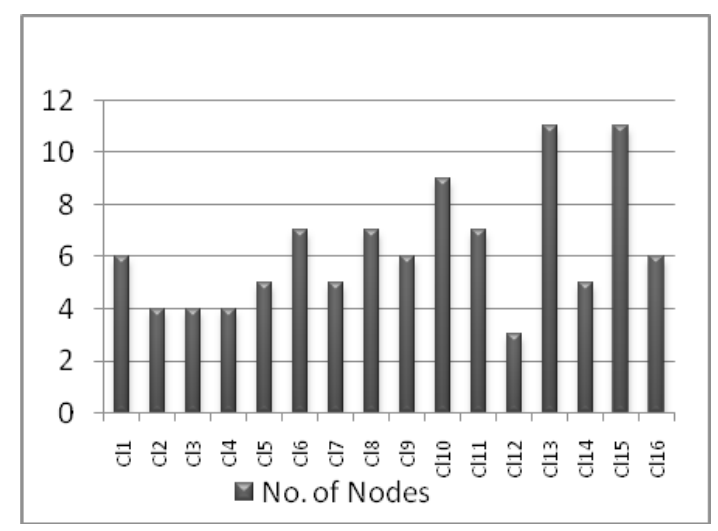

Figure 4. Unequal clustering in HUCA

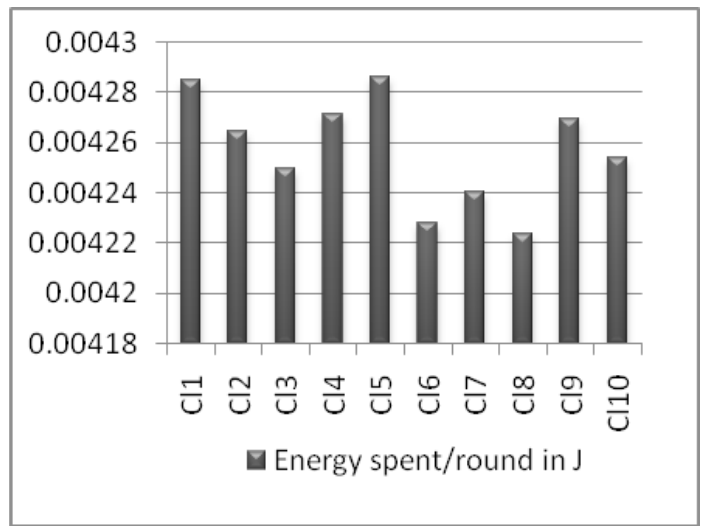

Figure 5. Energy consumption in LEACH. 


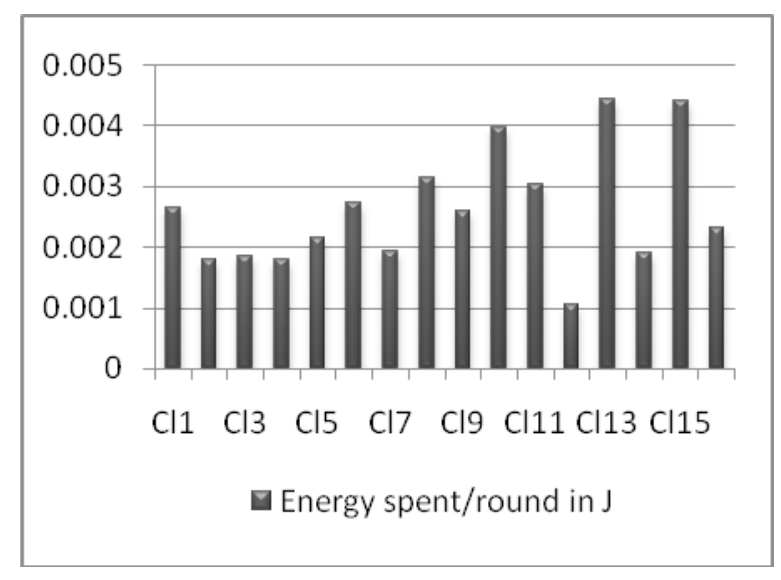

Figure 6. Energy consumption in HUCA.

\section{Conclusion}

The network area is divided into hierarchical layers and clusters are formed in each layer. Communication happens between $\mathrm{CH}$ nodes and a BS in a multi hop fashion which uses $\mathrm{CH}$ nodes as relay nodes. This proves more energy consumption when compared to the equal clustering LEACH protocol.

\section{References}

1. Liu T, Li Q, Liang P. An energy-balancing clustering approach for gradient-based routing in wireless sensor networks. Comput Comm. 2012; 35(17):2150-2161.

2. Chen J, Li Z, Kuo Y-H. A centralized balance clustering routing protocol for wireless sensor network. Wireless Person Communication. 2013; 72(1):623-634.
3. Hac A. Wireless sensor network designs. 5th ed. London: John Wiley and Sons, Ltd.; 2003.

4. Younis O, Fahmy S. HEED: a hybrid, energy-efficient, distributed clustering approach for Ad Hoc Sensor networks, IEEE Transactions on Mob Computing. 2004; 3(4):660-669.

5. Baiping L, Zhang X. Research and improvement of leach protocol for wireless sensor network [Lecture Notes]. International Conference on Information Engineering (ICIE); 2012.

6. Baranidharan B, Santhi B. EEGTP: Energy efficient graph theory protocol for wireless sensor networks. Inform Tech J. 2012; 11(7):808-811.

7. Kim J, Park S, Han Y, Chung T. CHEF: cluster head election mechanism using fuzzy logic in wireless sensor networks. Proceedings of the ICACT, 2008 Feb 17-20; Gangwon-Do; 2008. p.654-659.

8. Baranidharan B, Akilandeswari N, Santhi B. EECDC: Energy Efficient Coverage Aware Data Collection in Wireless Sensor Networks. Indian Journal of Science and Technology. 2013; 6(7):4903-4907.

9. Gupta I, Riordan D, Sampalli S. Cluster-head election using fuzzy logic for wireless sensor networks. Proceedings of the 3rd Annual Communication Networks and Services Research Conference; 2005 May 16-18. Canada: IEEE Computer Society; 2005. p. 255-260.

10. Bagci H, Yazici, A. An energy aware fuzzy approach to unequal clustering in wireless sensor networks. Applied Soft Computing. 2013; 13:1741-1749.

11. Mao S, Zhao C, Zhou Z, Ye Y. An improved fuzzy unequal clustering algorithm for wireless sensor network. Mobile Network Applications; 2013; 18(2):206-214.

12. Liao Y, Huan Q, Weiqun L. Load balanced clustering algorithm with distributed self-organization for wireless sensor networks. IEEE Sensor J; 2013; 13(5), 498-1506. 